九州大学学術情報リポジトリ

Kyushu University Institutional Repository

\title{
Monitoring of Geophysical Electromagnetic Environment for Space Weather Study - a Review
}

Yumoto, Kiyofumi

Faculty of Sciences, Kyushu University

https://doi.org/10.5109/1546838

出版情報：九州大学大学院理学研究院紀要：Series D, Earth and planetary sciences. 31 (3)， pp. 81-91, 2005-02-28. Faculty of Science, Kyushu University

バージョン：

権利関係 : 
Mem. Fac. Sci., Kyushu Univ., Ser. D, Earth \& Planet. Sci., Vol. XXXI, No. 3,

pp. 81-91, text-figs. 1-7, February, 2005

\title{
Monitoring of Geophysical Electromagnetic Environment for Space Weather Study - a Review
}

\author{
Kiyohumi Yumoto
}

\begin{abstract}
In order to understand impacts of electromagnetic environmental changes during severe geomagnetic storms and the magnetic hole in the Brazilian geomagnetically anomaly region on human technologies and ecosystems, coordinated satellite and groundbased observations are needed for Space Weather study. In this paper we will review how the geomagnetic induced currents and new radiation belt are formed during intense magnetic storms, and the magnetic hole affects on the earth's environment.
\end{abstract}

Keywords: electromagnetic environmental changes, geomagnetic induced currents, radiation belt, severe geomagnetic storms, the magnetic hole, Brazilian geomagnetic anomaly, space weather study

\section{I . Introduction}

The solar wind is charged particles streaming from the corona of the sun. The solar wind extends the sun's magnetic field into the solar system, and is known as the interplanetary magnetic field (IMF). The geomagnetic field of the earth interacts with the ever-changing interplanetary magnetic field. The charged particles of the solar wind streaming past the earth are deflected at the boundary of the interplanetary magnetic field and the earth's magnetic field, i.e. the magnetopause, generating electric current by a magneto-hydrodynamic process. The kinetic energy of solar wind particles is converted into electric energy producing more than a million megawatts of powers. The mechanism is called the solar-wind magnetosphere generator or auroral generator (Akasofu, 1968). The large injection of energy into the ionosphere by the auroral generator is through a pair of electric currents, called westward and eastward electrojets. These jet currents produce magnetic field variations on the ground.

Geomagnetic storms driven by variations in the solar wind can produce large changes in the geophysical electromagnetic environment and have significant impacts on human technologies. For example, spacecrafts are damaged by high-energetic charged particles striking solar cells and the lifetime of earth-orbiting satellites is shortened by enhanced orbital drag. The solar activity is also associated with long-term climate and environmental changes (Fris-Christensen and Lassen, 1991). During an intense geomagnetic storm, a new radiation belt for energies of several $10 \mathrm{MeV}$ is formed rapidly around $L=2.2$ in the inner magnetosphere (Blake et al., 1992, Li., et al., 1993, Obara et al., 2000). Magnetic disturbances can produce large geomagnetic induced currents (GIC's) in long conductors used for electrical power distribution, telecommunications, and pipelines on the earth's surface (Boteler et al,

Manuscript received on August 31, 2004 ; accepted on November 1, 2004. 
1989, Medford et al., 1989, Lanzerotti et al., 1992, Yumoto et al., 1999). A slowly changing potential gradients across the earth's surface produce quasi-dc currents, flowing through grounded ac transmission systems as a consequence (Boteler et al., 1989 and 1991).

On the other hand, high-energetic particles are precipitating in the magnetic hole of Brazilian geomagnetic anomaly region (Kohno et al., 1990), where the total field intensity is so weak and rapidly decreasing. The electric devices on board the satellite are reported to be damaged when it passes through the geomagnetic anomaly region. The intensity of radioactive rays excited by the highenergetic particles at micro-satellite altitude is 10,000 times as strong as the quantity we receive on the ground. Further studies are needed to investigate impacts of high energetic particle precipitation, and radioactive rays in the South Atlantic anomaly region on the atmospheric environment, human technologies and ecosystems.

In this paper, we will introduce changes of electromagnetic and energetic particle environment during intense geomagnetic storms and in the Brazilian geomagnetic anomaly region, and impacts on human technologies and ecosystems. Coordinated satellite and ground-based observations for Space Weather study will be very useful to understand global changes of the earth's electromagnetic environment and high-energetic particles, generated during intense geomagnetic storms and in the magnetic hole of the South American anomaly region.

\section{Geomagnetic induced currents during geomagnetic storms}

Gonzalez et al.(1990) investigated the solar cycle distribution of intense storms during cycles 20 and 21 , using values of the Dst index $<-100 \mathrm{nT}$ (nanotesra), and found dual-peak solar cycle distribution of intense geomagnetic storms. One peak occurs at the late ascending phase of the solar cycle or at solar maximum and another at the early descending phase of the cycle. The averaged separation of the peaks from solar maximum is about 8 months ahead for the first peak and about 25 months after for the second one. They suggested that the origin of the dual-peak distribution of the intense storms is associated with similar dual-peak distribution obtained for large-amplitude and long-duration vales of the negative $z$-component of the interplanetary magnetic field.

There are two types of geomagnetic storms; one occurs recurrently with 27days period, and the other occurs abruptly. The latter caused by transient events on the sun such as solar flares and coronal massive ejections (CME) is much severer than the former associated with the heliospheric structure of high-velocity stream and coronal hole. The power of the solar-

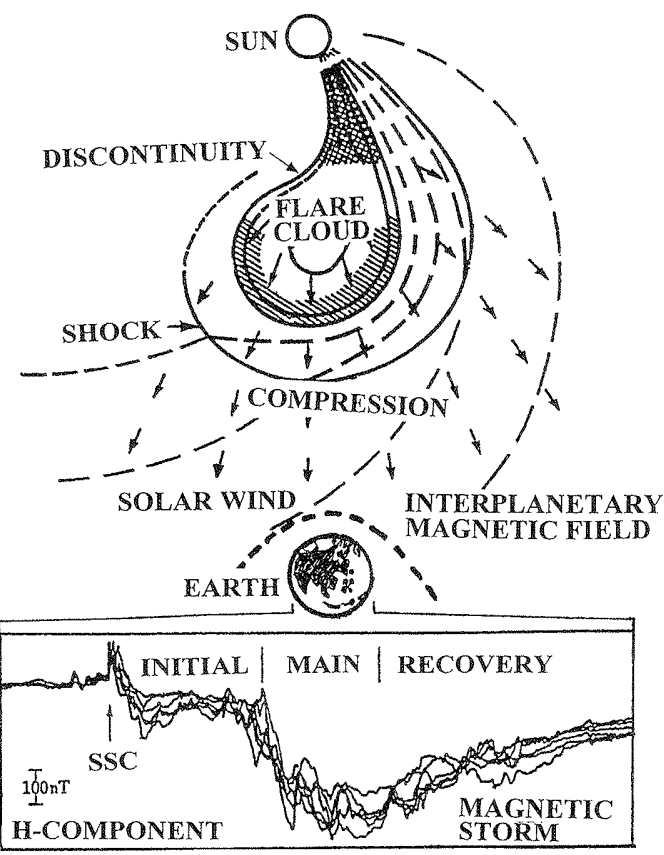

Fig. 1. A relationship of geomagnetic storms caused by the solar flare mass ejections. 
wind magnetosphere generator is ultimately controlled by transient events on the sun as shown in Figure 1. Shock waves are generated and propagate in the solar wind. The speed reaches 500 to 1,000 $\mathrm{km} / \mathrm{sec}$ just behind the wave front. As the shock wave collides with the earth's magnetic field, the power of the solar-wind magnetosphere generator can surge to 10 million megawatts or more, giving rise to a geomagnetic storm. Large-amplitude and long-duration negative z-component of the IMF enhances magnetospheric convection, which forms and intensifies ring currents in the inner magnetosphere, and then large deviations in magnetic fields are observed even at the lower latitudes and on the ground.

During a geomagnetic storm, magnetic disturbances in combination with enhanced ring current in the inner magnetosphere and ionospheric jet currents can affect both cable and "wireless" communications, such as trans-oceanic and trans-continental cables, oil pipelines, and long-haul power transmission lines. The influences of geomagnetic disturbances on long, man-made, earth-based conductors such as telegraph lines have been observed for well over a hundred years (see Lanzerotti and Medford, 1989). Medford et al.(1989) showed the cable power supply voltage at the North American end of the Fiber optic transatlantic telecommunications cable TAT-8 measured during the March 1989 storm, indicating the large scale changes as large as 700 volts in the total earth potential across the Atlantic, i.e. the potential gradient of as large as 0.12 volts $/ \mathrm{km}$ as shown in Figure 2. There were two intervals of time during which the true peak-to-peak voltage excursion over the course of a few to about twenty minutes was measured to be greater than 700 volts. Around 01:15 UT on March 14, the true rate of change of cross-Atlantic potential drop was of the order of 37 volts/min. Around 21:48 UT on March 13, the true rate of change of the potential drop was 300-450 volts/min. At this time, the rate of change of the magnetic field in the east-west component at Tuckerton was about $300 \mathrm{nT} / \mathrm{min}$. They concluded that the possible geomagnetic influences must be evaluated for the specific geomagnetic and geological locations and for the specific technologies used with the conductor system.
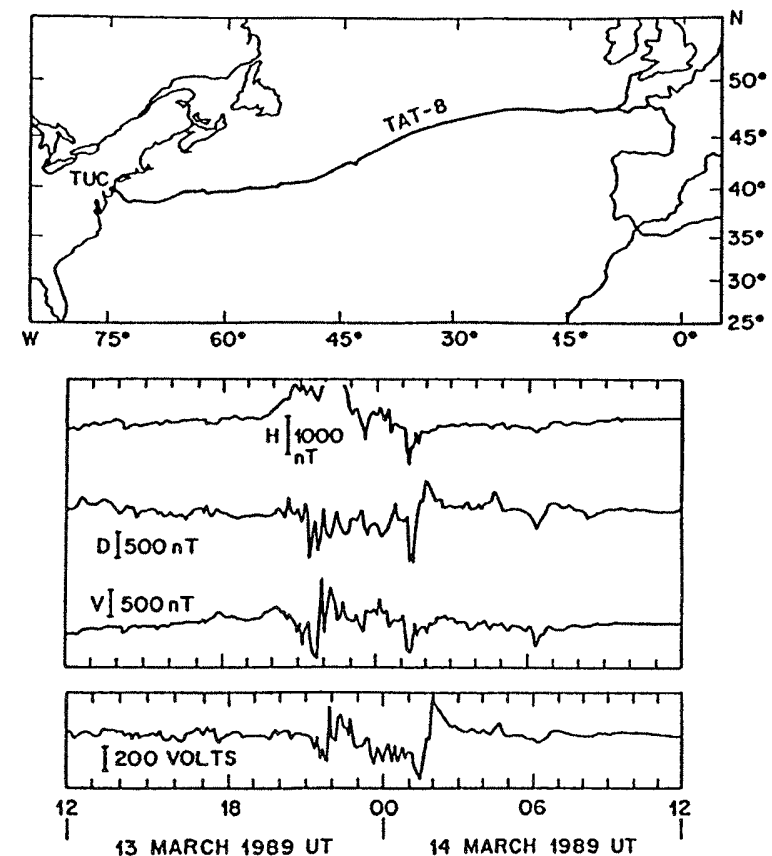

Fig. 2. (Top) Location of the TAT-8 transatlantic fiber optics telecommunications cable. (Middle, Bottom) Magnetic field and the cable voltage measured during the most intense phase of the geomagnetic storm during 13-14 March 1989 (Medford et al., 1989). 
The magnetic disturbances also induce currents in the earth's crust, causing earth surface potential gradients. The magnitude of such gradients is dependent not only on the intensities of magnetic storms and auroral substorms but the proximity to the magnetic poles and the resistivity of the ground. The earth surface potential can be created over large regions which can cause GIC to enter and exit power systems trough the transformer neutral grounding points. Because the auroral electrojets are in an eastwest direction and are a dominant influence in the induced earth currents, the earth surface potential is greatest in the east-west direction also. The east-west component of earth surface potential increases with increasing northern latitude as the auroral currents become more directly overhead.

On March 13-14, 1989, the earth experienced one of the worst geomagnetic storms in this century. The most serious effect of the storm occurred at 02:45 EST on March 13 when widespread geomagnetically induced currents (GIC) caused the saturation of transformers on the Hydro-Quebec power system (Boteler, et. al., 1989). The great magnetic storm of severe intensity induced a current of very low frequency in the Hydro-Quebec transmission system, then sustained a load of 21,350 MW, and knocked out power in almost all of Quebec, Canada, for about 5 hours. Figure 3 illustrates how magnetic disturbances, such as the severe geomagnetic storm of March 13, 1989 affect power systems (Boteler, et. al., 1991). The magnetic field variations (shown by the component H, D, and Z in Fig.3) induce electric field, Ex, in the ground; these drive geomagneticlly induced currents (GIC's) into power systems. The GIC's flow through power transformers, where they cause partial saturation of the transformer core by producing currents at $120 \mathrm{~Hz}, 180 \mathrm{~Hz}$, and higher harmonics of the power system frequency. The saturation of the transformers and the increased harmonic currents affect the stability of the system which, in extreme case, leads to a power blackout such as the one caused in Quebec by the March 1989 storm.

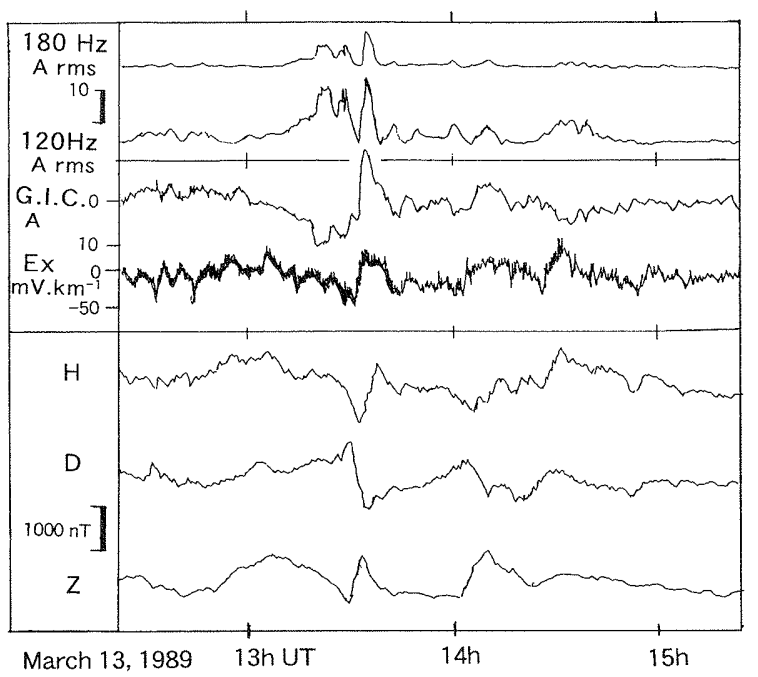

Fig. 3. During the severe geomagnetic storm of March 13, 1989, the magnetic field variations (H, D, and Z) induced electric fields, Ex, in the ground, geomagnetically induced currents (GIC's) into power system. The GIC's flow through power transformers, where they produce currents at $120 \mathrm{~Hz}, 180 \mathrm{~Hz}$, and higher harmonics of the power system frequency (Boteler et al., 1991). 


\section{New formation of radiation belt during intense magnetic storms}

The typical spatial distribution of certain class of radiation-belt particles (viz. electrons having kinetic energies $\mathrm{E}>0.5 \mathrm{MeV}$ ) shows a two-zone structure as shown in Figure 4. The most intense radiation occurs in the inner zone at $\mathrm{L}<2$, but there is typically a secondary maximum between $\mathrm{L}=4$ and 6 (the outer zone). The so-called slot region $(2<\mathrm{L}<4)$ contains a relative minimum in radiation intensity and serves to separate the two radiation zones from each other. The two-zone structure characteristic of geomagnetically trapped electron radiation can be understood qualitatively in terms of an unsteady filling of the trapping region from outside (see Schulz, 1991). The quiet-time slot region seems to become "filled" with electrons during a magnetic storm, but tends to lose its energetic electrons within 5-10 days thereafter. This is in contrast to the inner radiation zone, where the maximum lifetime (about 300 days at $\mathrm{L}=1.5$ ) roughly coincides with the peak in radiation intensity.

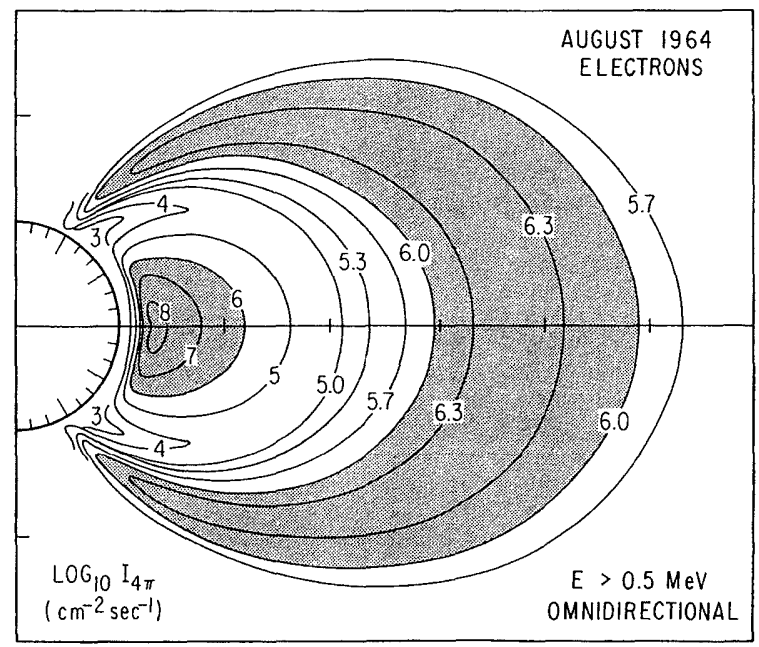

Fig. 4. Contours map of constant integral electron flux in the earth's radiation environment. Shaded regions correspond to inner and outer radiation belts (Vette et al., 1966).

The rapid formation of new proton and electron radiation belts was firstly reported by Blake et al.(1992) using the CRESS satellite observations at $\mathrm{L}=2.5$ during the March 24, 1991 storm event. The new proton radiation belt for 20 to $80 \mathrm{MeV}$ energy was found to be formed around $\mathrm{L}=2.4$, while the new electron radiation belt for more than $15 \mathrm{MeV}$ energy was formed around $\mathrm{L}=2.2$. The prompt energization and transport of radiation belt particles during the storm sudden commencement were simulated by an impulsive electric field variation (Li., et al, 1993, Hudson, et al., 1997). Obara et al. (2001) speculated that substorm-related electric and magnetic field fluctuations and/or waves would accelerate electron efficiently. They demonstrated a case study, showing that the internal acceleration is actually taking place and it leads a large enhancement of the relativistic electrons in the outer radiation belt.

A severe geomagnetic storm driven by the fast solar wind (averaged speed at $1 \mathrm{AU}$ was more than $900 \mathrm{~km} / \mathrm{sec}$ with $-54 \mathrm{nT}$ of IMF/Bz) began with an ssc ( $\Delta \mathrm{H}=140 \mathrm{nT}$ at Kakioka) at 14:36 UT on July 15 , 2000; developed rapidly from 20:00 UT for the next 4 hours; and reached a minimum Dst of $-295 \mathrm{nT}$ around 00:00 UT. Obara et al.(2001) showed the relationship among L-t diagram for the electrons with 
energy from $300 \mathrm{KeV}$ to $1.1 \mathrm{MeV}, \mathrm{Kp}$ index and Dst index variations for the interval from July 11 to 20 , 2000. In the energetic particle data provided by NOAA and Akebono satellites, rapid formation of new radiation belts for $0.3-1.1 \mathrm{MeV}$ electron energy can be seen around $\mathrm{L} \sim 2.6$ and 4.0 just after the onset of the ssc storm (at 14:36 UT on July 15, 2000). These high-energetic particles from the newly formed radiation belts must be precipitating in the Brazilian geomagnetic anomaly region, where the total field intensity is so weak and rapidly decreasing as described in the next session.

On the other hand, significant factor that may influence the functioning of space systems is the solar proton events (SPE). The bursts of protons and ions with high energies (up to $1 \mathrm{GeV}$ ) accompany solar flares, and occur more frequently at the maximum of solar activity. Solar cosmic rays penetrate throughout the magnetosphere and can cause single event upsets, degradation of solar panels, etc.

Serious satellite anomalies, even total failures of spacecraft, can be caused by relativistic ("killer") electrons in the outer radiation belt (Wrenn, et al., 2000). Contrary to the solar protons, the "killer" electrons are energized locally by waves within the magnetosphere during a geomagnetic storm. The acceleration of electrons due to the substorm injection is more effective as pre-process. One of the main mechanisms of the acceleration and inward diffusion is probably related to the drift-resonant interaction of electrons with large-scale ultra-low-frequency (ULF) waves (Elkington et al., 1999, Liu et al., 1999). These waves are formed by resonant filtering and amplification from wide-band magnetospheric MHD noise upon conversion into field line Alfve'n oscillations.

Rather surprisingly, not the magnetic storm intensity (as measured by Dst index), but the presence of Pc5 (150-600 s) ULF power after minimum Dst is happened to be a good indicator of relativistic electron response. To characterize a global ULF activity O'Brien et al. (2001) constructed a simple hourly "ULF wave index", characterizing the ground spectral power in the Pc5 band at any LT and L=3.5-7.0. It was shown that among basic solar wind and magnetospheric parameters, the long duration Pc5 wave power during the recovery phase appeared to discriminate best between those storms that do and do not produce relativistic electrons. Similarly, storms with substantial electron enhancements were found to have a higher ULF wave power by about an order of magnitude in the recovery phase of magnetic storms (Mathie and Mann, 2001). Thus, in a laminar non-turbulent magnetosphere the "killer" electrons probably would not appear.

\section{Effect of the magnetic hole on the earth's environment}

The geomagnetic field is monotonically decreasing over the last 1,000 years not only in Brazil but also all over the world (Fraser-Smith, 1987). Peddie (1982) showed the annual change of total geomagnetic field intensity. The peak field intensities lie at the Northern polar region near Canada and the Southern polar region near the Antarctic continent facing Australia as shown in Figure 5 (Takeda et al., 1993). The total intensity of the geomagnetic field in the polar region exceeds $60,000 \mathrm{nT}$. On the other hand, the total intensity of geomagnetic field in Brazil is only $23,000 \mathrm{nT}$. This value is abnormally weak as compared with the one in other mid-latitude region. This region is called South Atlantic Geomagnetic Anomaly, Brazilian Geomagnetic Anomaly, or Geomagnetic hole (Makita and Schuch, 1999).

The geomagnetic field around the Brazilian anomaly region is rapidly decreasing and the size of the anomaly region is expanding year by year. For example, in the Caribbean Sea, the South Atlantic Ocean and the Antarctic Peninsula, the rate of decrease of the geomagnetic field exceeds $100 \mathrm{nT}$ per year. Near the center of the Brazilian anomaly region, the rate of decrease of the geomagnetic field is not so large (about $40 \mathrm{nT}$ per year) but its intensity is steadily decreasing. Conversely, the intensity of the geomagnetic field in the Japanese archipelago and the Eurasian Continent is increasing slightly. Gubbins (1987) proposed a hypothesis that a localized reverse magnetic field is appearing under Brazil region, and 


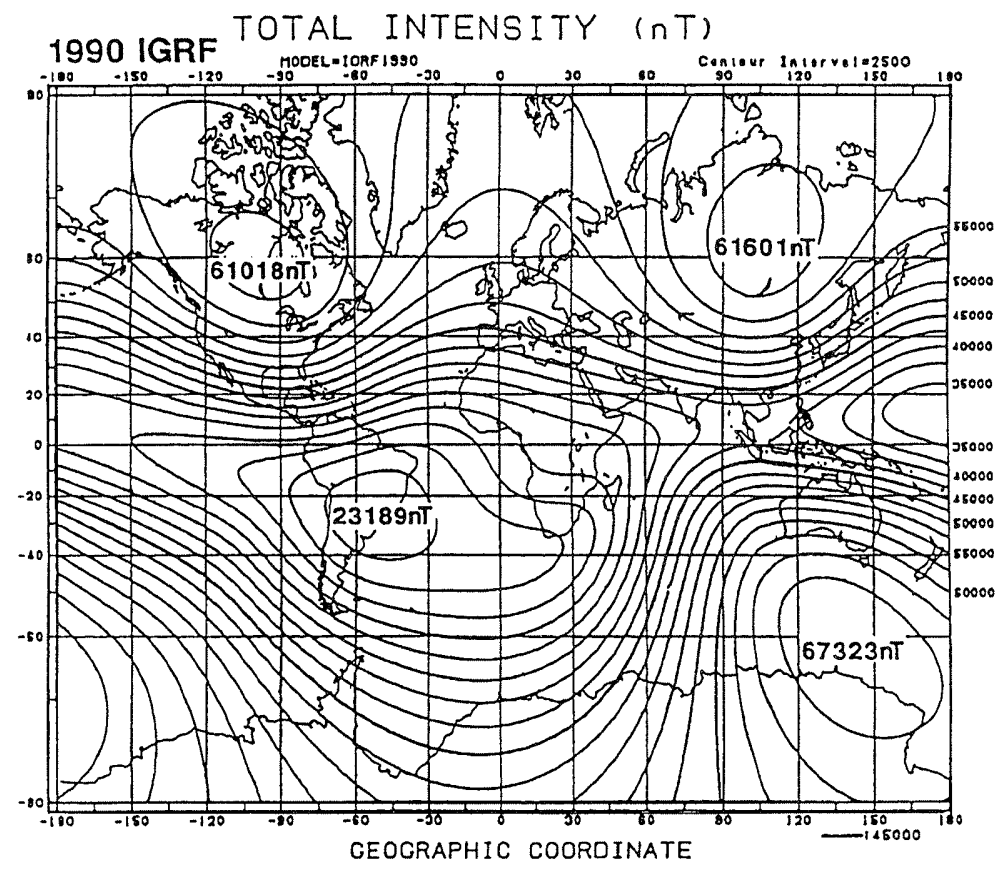

Fig, 5. Geomagnetic field total force (nT) of the 1990 IGRF. The total intensity of the geomagnetic field exceeds $60,000 \mathrm{nT}$ in the polar regions, while it is only 23,000 nT in the South Atlantic anomaly region (After Takeda et al., 1993).

weakens the ambient dipole field component in the region. He named this localized magnetic field as "Core-Spot", which is located in the earth's outer core. If the Core-Spot increases in its intensity and expands world-wide, polarity reversal of the geomagnetic field can be expected to occur in the near future. If the rate of decrease of the geomagnetic field continues, the geomagnetic field around the Brazilian anomaly region will disappear in less than 500 years. A period of 500 years is very short from a geological standpoint. Human beings may have to deal with circumstances in a very weak geomagnetic field. It is needed to study what kind of effects of the decreasing geomagnetic field on the earth's environment will happen in near future.

A new space age began when the first satellite was launched by USSR in 1957. Four decades on our knowledge about space have advanced rapidly through extensive satellite observations. We have come to understand that a multitude of electrons and protons precipitate into the polar region and excite aurora (see Akasofu, 1968). In addition it became clear that a lot of high-energy particles are precipitating into Brazil and the South Atlantic Ocean. For each particle gyrating about a given field line, there is a mirror-point field $(\mathrm{Bm})$ where the particle conserves the first adiabatic invariant ( $\mathrm{mv} \perp^{2} / 2 \mathrm{~B}$ ) and reverses the movement along the line of force. If the field line actually contains the so-called mirror points above the top of the atmosphere (about $100 \mathrm{~km}$ ), the particle will literally be trapped between the mirror points. But, the total intensity of geomagnetic field in the Brazilian Geomagnetic Anomaly region is only $23,000 \mathrm{nT}$, which is abnormally weak as compared with that (about 35,000 nT) at the conjugate point in the northern hemisphere. The particles which can reverse their movements at the mirror-point 
field of Bm=23,000-35,000 nT above the top of the northern atmosphere, however, are said to be in the loss cone and precipitate into the southern anomaly region.

According to the OHZORA satellite observations as shown in Figure 6, the flux of precipitating particles at altitude $700-850 \mathrm{~km}$ in the South Atlantic anomaly region is 1,000 times higher than that in other region (Kohno et al., 1990). The precipitating electron and proton energy are higher than those in the auroral region, and therefore X-ray emission occurs in this region. Due to the significant amount of high-energy particles precipitating over the Brazilian anomaly region, electric devices on board the satellites are affected. For example, problems with semi-conductor memory are reported to occur frequently in the Brazilian anomaly region as shown in Figure 7. High-energy particles sometimes damage the measuring system on board the satellites, so several devices were switched off while passing through this area.
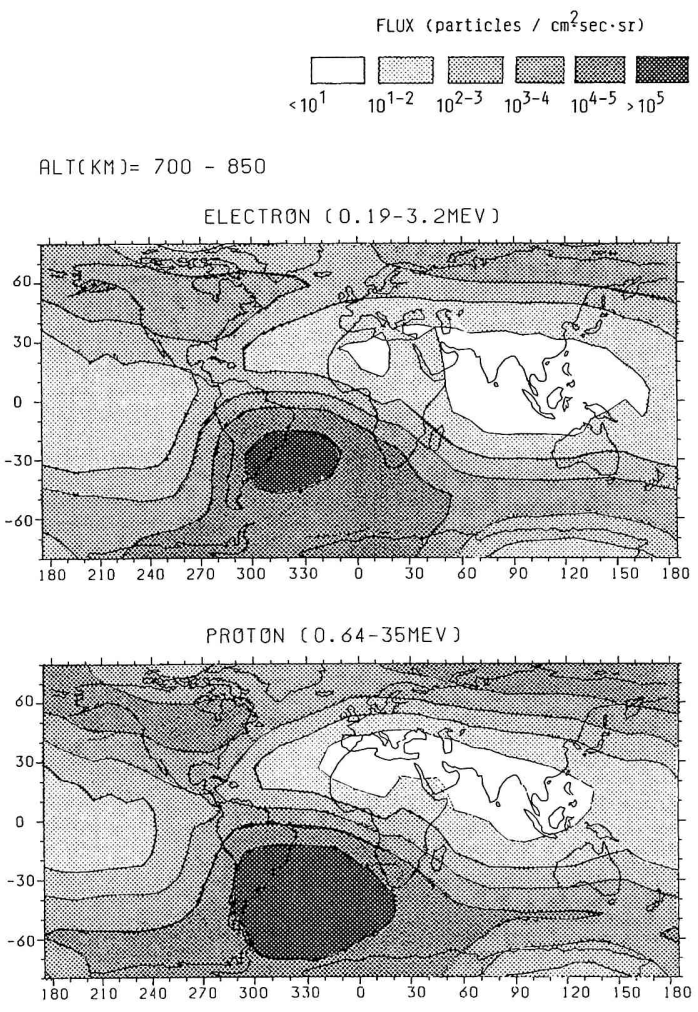

Fig. 6. Global distributions of electron (Top) and proton (Bottom) observed by OHZORA at altitude 700-850 $\mathrm{km}$ from February 1984 to January 1987. A large number of electrons and protons are precipitating over the South Atlantic anomaly region (After Kohno et al., 1990).

It is also reported that measuring of X-rays propagating from a black hole star is difficult in the Brazilian geomagnetic anomaly region, because X-rays caused by high-energy particles in this region interfere with the measurement. Recently, the Russian satellite has been detecting radioactive rays emitted by high-energy particles. The peak intensity of these radioactive rays coincides with the peakarea of precipitating particle flux observed by the OHZORA and NOAA satellites. Fujitaka (1998) 


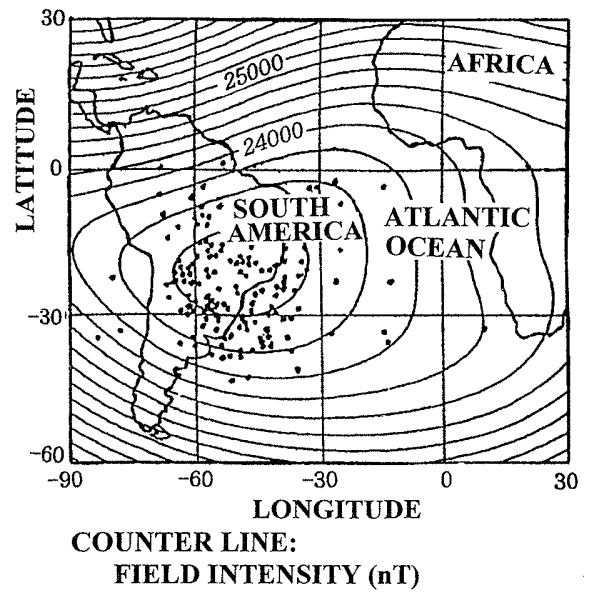

Fig. 7. Occurrence distribution of semi-conductor memory problems of multiple satellites around the Brazilian geomagnetic anomaly region.

estimated that the intensity of radioactive rays in this area reaches to $0.69 \mathrm{mGy}$. The intensity of radioactive rays at this altitude is 10,000 times as strong as the quantity we receive on the ground. Further studies by low altitude micro-satellites, e.g., DEMETER, are needed to investigate impacts of high energetic particle precipitations, and radioactive rays in the South Atlantic anomaly region on the atmospheric environment, human technologies and the ecosystems.

\section{Conclusion}

In this paper we reviewed severe impacts of the geomagnetic induced currents (GIC's) and new radiation belt during intense magnetic storms, and high-energetic particles in the magnetic hole on the human technologies and ecosystems. Geomagnetic storms driven by variations in the solar wind can produce large changes in the geophysical electromagnetic environment and have significant impacts on human technologies. For example, spacecrafts are damaged by high-energetic charged particles striking solar cells and the lifetime of earth-orbiting satellites are shortened by enhanced orbital drag. During an intense geomagnetic storm, a new radiation belt for energies of several $10 \mathrm{MeV}$ is also formed rapidly around $\mathrm{L}=2.2$ in the inner magnetosphere. Magnetic disturbances produce large GIC's in long conductors used for electrical power distribution, telecommunications, and pipelines on the earth's surface. A slowly-changing potential gradients across the earth's surface produces quasi-dc currents, flowing through grounded ac transmission systems as a consequence. On the other hand, high-energetic particles are precipitating in the magnetic hole of Brazilian geomagnetic anomaly region, where the total field intensity is so weak and rapidly decreasing. The electric devices on board the satellite are damaged when it passes through the geomagnetic anomaly region. The intensity of radioactive rays excited by the high-energetic particles at micro-satellite altitude is 10,000 times as strong as the quantity we receive on the ground.

It is concluded that further coordinated satellite and ground-based observations for Space Weather studies are needed to investigate impacts of GIC's, high energetic particle precipitation, and radioactive rays in the South Atlantic anomaly region on the atmospheric environment, human technologies and ecosystems. 


\section{Acknowledgments}

The author would like to express his sincere thanks to Drs. K. Makita and T. Obara for their useful comments on this review paper.

\section{References}

Akasofu, S.-I. (1968) Polar and Magnetospheric Substorms. Astrophysics and Space Science Library, D. Reidel Publ. Comp., Dordrecgt-Holland, 280pp.

Blake, J.B., W.A. Kolosinski, R.W. Fillius and E.G. Mullen (1992) Injection of electrons and protons with energies of tens of MeV into L $<3$ on 24 March 1991. Geophys. Res. Lett. 19, 821-825.

Boteler, D.H., R.M. Shier, T. Watanabe and R.E. Horita (1989) Effects of geomagneticlly induced currents in the B.C. Hydro $500 \mathrm{kV}$ system. IEEE Trans. Power Delivery, 4, 818-823.

Boteler, D.H., T. Watanabe, D.B. Butler, R.E. Horita and G.M. Chen (1991) Prediction of geomagnetically induced current levels in the B.C. Hydro $500 \mathrm{kV}$ system. Solar-Terr. Predict. Proc., 2, 30-37.

Elkington, S.R., M.K. Hudson and A.A. Chan (1999) Acceleration of relativistic electrons via driftresonant interaction with toroidal-mode Pc5 ULF oscillations. Geophys. Res. Lett., 26, 3,2733,276 .

Fraser-Smith, A.C.(1987) Centered and eccentric geomagnetic dipoles and their poles, 1600-1985. Rev. Geophys., 25, 1-16.

Fris-Christensen E. and K. Lassen (1991) Length of the solar cycle: An indicator of solar activity closely associated with climate. Science, 254, 698-700.

Fujitaka, K.(1998) Environmental changes of radioactive rays in space and on the Ground. "Gekkan Chikyu", Suppl., No. 22, 26-34 (in Japanese).

Gonzalez, W.D., A.L.C. Gonzalez and B.T. Tsurutani (1990) Dual-peak solar cycle distribution of intense geomagnetic storms. Planet Space Sci., 38, 181-187.

Gubbins, D.(1987) Mechanism for geomagnetic reversals, Nature, 326, 167-168.

Hudson, M.K., S.R. Elkington, J.G. Lyon, V.A. Marchenko, I. Roth, M. Temerin, J.B. Blake, M.S. Gussenhoven and J. R. Wygant (1997) Simulation of radiation belt formation during storm sudden commencements. J. Geophys. Res., 102, 14,087-14,102.

Kohno, T., K. Munakata, K. Nagata, H. Murakami, A. Nakamoto, N. Hasebe, J. Kikuchi and T. Doke (1990) Intensity maps of $\mathrm{MeV}$ electrons and protons below the radiation belt. Planet. Space Sci., 38, 483-490.

Lanzerotti, L.J. and L.V. Medford (1989) Geomagnetic disturbances and long-haul telecommunications cables. Proc. EPRI Conference on Geomagnetically-Induced Currents, held on Nov. 8-10, 1989 at San Francisco, California, 1-4.

Lanzerotti, L.J., L.V. Medford, J.S. Kraus, C.G. Maclennan and R.D. Hunsucker (1992) Possible measurements of small-amplitude TID's using parallel unpowered telecommunications cables. Geophys. Res. Lett., 19, 253-256.

Li, X., M. Roth, M. Temerin, J.R. Wygant, N.K. Hudson and J.B. Blake (1993) Simulation of the prompt energization and transport of radiation belt particles during the March 24, 1991 ssc. Geophys. Res. Lett., 20, 2,423-2,426.

Liu, W.W., G. Rostoker and D.N. Baker (1999) Internal acceleration of relativistic electrons by largeamplitude ULF pulsations. J. Geophys. Res., 104, 17391-17407.

Makita, K. and N.J. Schuch (1999) Geomagnetic hole, Bull. Sci. Engin., Takushoku Univ., 7, 59-66. 
Mathie, R.A. and I.R. Mann (2001) On the solar wind control of Pc5 ULF pulsation power at mid-latitudes: Implications for $\mathrm{MeV}$ electron acceleration in the outer radiation belt. J. Geophys. Res., 106, 29,78329,796 .

Medford, L.V., L.J. Lanzerotti, J.S. Kraus and C.G. Maclennan (1989) Transatlantic Earth potential variations during the March 1989 magnetic storms. Geophys. Res. Lett., 16, 1145-1148.

Obara, T. M. Den, Y. Miyoshi and A. Morioka (2000) Energetic electron variation in the outer radiation zon during early May 1998 magnetic storm. J. Atmos. Solar-Terr. Phys., 63, 1407-1412.

Obara, T., M. Den,T. Nagatsuma and X. Li (2000) Formation of new electron radiation belt during magnetic compression event. Advances in Space Research, (Proceeding of COSPAR Meeting in Poland, 2000)

Obara, T., Y. Miyoshi and A. Morioka (2001) Large enhancement of the outer belt electrons during magnetic storms. Earth Planets Space, 53, 1163-1170.

O'Brien, T.P., R.L. McPherron, D. Sornette, G.D. Reeves, R. Friedel and H.J. Singer (2001) Which magnetic storms produce relativistic electrons at geosynchronous orbit? J. Geophys. Res., 106, $15,533-15,544$.

Peddie, N.W.(1982) International geomagnetic reference field: The third generation. J. Geomag. Geoelectr., 34, 309-326

Schulz, M.(1991) The Magnetosphere in Geomagnetism Vol. 4 edited by J.A. Jacobs, Academic Press Limited, 87-293pp.

Takeda, M., T. lyemori and T. Kamei (1993) Data Catalogue, World Data Center C 2 Geomagnetic Data book, 23, 175pp.

Vette, J.I., A.B. Lucero and J.A. Wright (1966) Inner and Outer Zone Electrons. NASA SP-3024, Washington, D.C.

Wrenn, G.L., D.J. Rodgers and P. Buehler (2000) Modeling the outer belt enhancements of penetrating electrons, J. Spacecraft, 37, 408-417.

Yumoto, K., H. Utada and Y. Hamano (1999) Geomagnetically induced currents in the weatern Pacific region during February 8-9, 1992 magnetic storm. Mem. Fac., Sci., Kyushu Univ., Ser. D, Earth \& Planet. Sci., 30, 111-118. 